\title{
MIXED NORMS AND SOBOLEV TYPE INEQUALITIES
}

\author{
V. I. KOLYADA \\ Department of Mathematics, Karlstad University \\ Universitetsgatan 1, 65188 Karlstad, Sweden \\ E-mail: viktor.kolyada@kau.se
}

\begin{abstract}
We study mixed norm spaces that arise in connection with embeddings of Sobolev and Besov spaces. We prove Sobolev type inequalities in terms of these mixed norms. Applying these results, we obtain optimal constants in embedding theorems for anisotropic Besov spaces. This gives an extension of the estimate proved by Bourgain, Brezis and Mironescu for isotropic Besov spaces.
\end{abstract}

1. Introduction. Let $1 \leq p<\infty$. Denote by $W_{p}^{1}\left(\mathbb{R}^{n}\right)$ the Sobolev space of all functions $f \in L^{p}\left(\mathbb{R}^{n}\right)$ for which every first-order weak derivative $\partial f / \partial x_{k} \equiv D_{k} f$ exists and also belongs to $L^{p}\left(\mathbb{R}^{n}\right)$. The classical Sobolev theorem asserts that for any function $f$ in $W_{p}^{1}\left(\mathbb{R}^{n}\right)(1 \leq p<n)$

$$
\|f\|_{q^{*}} \leq c \sum_{k=1}^{n}\left\|D_{k} f\right\|_{p}, \quad q^{*}=\frac{n p}{n-p} .
$$

Sobolev proved this inequality in 1938 for $p>1$; his method, based on integral representations, did not work in the case $p=1$. Only at the end of fifties Gagliardo and Nirenberg gave simple proofs of the inequality (1.1) for all $1 \leq p<n$. The central part of Gagliardo's approach [6] was the following lemma.

LEMmA 1.1. Let $n \geq 2$. Assume that $g_{k} \in L^{1}\left(\mathbb{R}^{n-1}\right) \quad(k=1, \ldots, n)$ are non-negative functions on $\mathbb{R}^{n-1}$. Then

$$
\int_{\mathbb{R}^{n}}\left(\prod_{k=1}^{n} g_{k}\left(\hat{x}_{k}\right)\right)^{1 /(n-1)} d x \leq\left(\prod_{k=1}^{n} \int_{\mathbb{R}^{n-1}} g_{k}\left(\hat{x}_{k}\right) d \hat{x}_{k}\right)^{1 /(n-1)} .
$$

2000 Mathematics Subject Classification: Primary 46E30, 46E35.

Key words and phrases: mixed norms, rearrangements, embeddings, Sobolev spaces, Besov spaces.

This research was partially supported by grant BFM2003-06335-C03-02 of the DGI, Spain.

The paper is in final form and no version of it will be published elsewhere. 
As usual, for any vector $x \in \mathbb{R}^{n}$ we denote by $\hat{x}_{k}$ the $(n-1)$-dimensional vector obtained from $x$ by removal of its $k$ th coordinate.

Assume that $f \in W_{1}^{1}\left(\mathbb{R}^{n}\right)$. Then for almost all $x \in \mathbb{R}^{n}$ and every $k=1, \ldots, n$

$$
|f(x)| \leq \frac{1}{2} \int_{\mathbb{R}}\left|D_{k} f(x)\right| d x_{k} \equiv \frac{1}{2} g_{k}\left(\hat{x}_{k}\right) .
$$

Thus, applying (1.2), we immediately obtain the inequality

$$
\|f\|_{n /(n-1)} \leq \frac{1}{2}\left(\prod_{k=1}^{n}\left\|D_{k} f\right\|_{1}\right)^{1 / n} .
$$

This yields (1.1) for $p=1$.

However, a stronger statement can be derived from (1.2). Let

$$
V_{k} \equiv L_{\hat{x}_{k}}^{1}\left(\mathbb{R}^{n-1}\right)\left[L_{x_{k}}^{\infty}(\mathbb{R})\right] \quad(1 \leq k \leq n)
$$

be a space with the mixed norm

$$
\|f\|_{V_{k}} \equiv \int_{\mathbb{R}^{n-1}} \varphi_{k}\left(\hat{x}_{k}\right) d \hat{x}_{k}
$$

where

$$
\varphi_{k}\left(\hat{x}_{k}\right)=\operatorname{ess} \sup _{x_{k} \in \mathbb{R}}|f(x)| .
$$

Gagliardo's lemma immediately implies the following theorem.

TheOREM 1.2. Assume that $f \in \cap_{k=1}^{n} V_{k}, \quad n \geq 2$. Then $f \in L^{n /(n-1)}\left(\mathbb{R}^{n}\right)$ and

$$
\|f\|_{n /(n-1)} \leq\left(\prod_{k=1}^{n}\|f\|_{V_{k}}\right)^{1 / n} \text {. }
$$

Since for $f \in W_{1}^{1}\left(\mathbb{R}^{n}\right)$

$$
\|f\|_{V_{k}} \leq \frac{1}{2}\left\|D_{k} f\right\|_{1} \quad(k=1, \ldots, n),
$$

then (1.3) follows from Theorem 1.2.

Denote by $f^{*}$ the non-increasing rearrangement of a measurable function $f$. If $1 \leq$ $q, p<\infty$, then the Lorentz space $L^{q, p}\left(\mathbb{R}^{n}\right)$ is defined as the class of all measurable functions $f$ on $\mathbb{R}^{n}$ such that

$$
\|f\|_{q, p} \equiv\left(\int_{0}^{\infty}\left(t^{1 / q} f^{*}(t)\right)^{p} \frac{d t}{t}\right)^{1 / p}<\infty .
$$

For any fixed $q$, the Lorentz spaces increase as the secondary index $p$ increases (see Section 2 below).

It is well known that the left-hand side in (1.1) can be replaced by the stronger $L^{q^{*}, p}$-Lorentz norm. That is, there holds the inequality

$$
\|f\|_{q^{*}, p} \leq c \sum_{k=1}^{n}\left\|D_{k} f\right\|_{p} \quad\left(1 \leq p<n, \quad q^{*}=\frac{n p}{n-p}\right)
$$

(see [20], [21], [22]). For $p>1$ this result can be obtained by interpolation (although the direct proof is simpler). There are numerous proofs of (1.6) in the case $p=1$; most of them are related to rearrangements, properties of level sets, and geometric inequalities. 
A very interesting approach given by Fournier [8] was based on the following refinement of the Theorem 1.2.

Let $V_{k}$ be the spaces defined by (1.4).

Theorem 1.3. Assume that $f \in \cap_{k=1}^{n} V_{k}, \quad n \geq 2$. Then $f \in L^{n /(n-1), 1}\left(\mathbb{R}^{n}\right)$ and

$$
\|f\|_{n /(n-1), 1} \leq\left(\prod_{k=1}^{n}\|f\|_{V_{k}}\right)^{1 / n} .
$$

Taking into account (1.5), we immediately obtain (1.6) for $p=1$. More exactly, we obtain the following refinement of the inequality (1.3)

$$
\|f\|_{n /(n-1), 1} \leq \frac{1}{2}\left(\prod_{k=1}^{n}\left\|D_{k} f\right\|_{1}\right)^{1 / n} .
$$

Thus, inequality (1.8) (as well as (1.3)) can be broken down into two successive steps. The main step is the inequality (1.7). To derive (1.8) from (1.7) we have only to apply the following simple fact: if a function $f \in L^{1}\left(\mathbb{R}^{n}\right)$ has a weak derivative $D_{k} f \in L^{1}\left(\mathbb{R}^{n}\right)$, then $f \in V_{k}($ see $(1.5))$.

One of our main problems in this paper is to find an analogue of Theorem 1.3 for more general mixed norm spaces. To clarify this problem we can consider the following example. Let $n=2$ and $1 \leq r<\infty$. Assume that

$$
f \in L_{y}^{1}(\mathbb{R})\left[L_{x}^{r}(\mathbb{R})\right] \quad \text { and } \quad f \in L_{x}^{1}(\mathbb{R})\left[L_{y}^{r}(\mathbb{R})\right] .
$$

Which Lorentz space does the function $f$ belong to?

First of all, we study mixed norm spaces related to the Sobolev spaces $W_{p}^{1}$ and inequality (1.6) for arbitrary $1 \leq p<n$. We have seen that if $D_{k} f \in L^{1}\left(\mathbb{R}^{n}\right)$ for some $k$, then $f \in V_{k} \equiv L_{\hat{x}_{k}}^{1}\left[L_{x_{k}}^{\infty}\right]$. Suppose now that $D_{k} f \in L^{p}\left(\mathbb{R}^{n}\right)$ for some $p>1$; what is the corresponding space $V_{k}$ in this case? A similar question arises if a function $f$ belongs to a Besov space with respect to a separate variable $x_{k}$. In turn, this question is related to embeddings of anisotropic Besov spaces.

Studying these problems, we introduce a scale of generalized spaces with mixed norms similar to the spaces $V_{k}$. In particular, the spaces

$$
L^{p}\left(\mathbb{R}^{n-1}\right)\left[L^{r, \infty}(\mathbb{R})\right] \quad(1 \leq p, r<\infty)
$$

are contained in this scale. ${ }^{1}$ First we define "weak" spaces $\Lambda^{\sigma}$.

Let $\sigma \in \mathbb{R}$. Denote by $\Lambda^{\sigma}(\mathbb{R})$ the space of all measurable functions $f$ such that

$$
\|f\|_{\Lambda^{\sigma}} \equiv \sup _{t>0} t^{\sigma}\left[f^{*}(t)-f^{*}(2 t)\right]<\infty .
$$

If $0<\sigma<\infty$ and $r=1 / \sigma$, then $\Lambda^{\sigma}(\mathbb{R})=L^{r, \infty}(\mathbb{R})$. If $\sigma=0$, then $\Lambda^{\sigma}$ coincides with the space weak- $L^{\infty}$ introduced in [2]. If $\sigma<0$, then (1.9) is a weak version of Lipschitz condition for the rearrangement $f^{*}$.

The main result of this paper (Theorem 3.1), in particular, states the following.

\footnotetext{
${ }^{1} L^{r, \infty}$ is the space of all measurable functions $f$ such that $\sup _{t>0} t^{1 / r} f^{*}(t)<\infty$.
} 
Theorem 1.4. Assume that $1 \leq p<\infty, n \geq 2(n \in \mathbb{N})$, and that $\alpha_{k}(k=1, \ldots, n)$ are positive numbers such that

$$
\alpha \equiv n\left(\sum_{k=1}^{n} \frac{1}{\alpha_{k}}\right)^{-1} \leq \frac{n}{p} .
$$

Let

$$
\sigma_{k}=\frac{1}{p}-\alpha_{k} \quad \text { and } \quad V_{k} \equiv L_{\hat{x}_{k}}^{p}\left(\mathbb{R}^{n-1}\right)\left[\Lambda_{x_{k}}^{\sigma_{k}}(\mathbb{R})\right]
$$

Then

$$
\bigcap_{k=1}^{n} V_{k} \subset L^{q^{*}, p}\left(\mathbb{R}^{n}\right), \quad q^{*}=\frac{n p}{n-\alpha p} .
$$

Observe that we get the optimal constant in the corresponding estimate of the Lorentz norm in (1.10). The limiting case $\alpha=n / p$ also is included (the definition of $L^{\infty, p}$ is given in the Section 2).

It can be easily proved that if a function $f \in L^{p}\left(\mathbb{R}^{n}\right) \quad(1 \leq p<\infty)$ has a weak derivative $D_{k} f \in L^{p}\left(\mathbb{R}^{n}\right)$, then $f \in L_{\hat{x}_{k}}^{p}\left(\mathbb{R}^{n-1}\right)\left[\Lambda_{x_{k}}^{1 / p-1}(\mathbb{R})\right]$ (see Proposition 3.4 below). Thus, taking $\alpha_{1}=\cdots=\alpha_{n}=1$ and applying (1.10), we immediately get the embedding

$$
W_{p}^{1}\left(\mathbb{R}^{n}\right) \subset L^{q^{*}, p}\left(\mathbb{R}^{n}\right), \quad q^{*}=\frac{n p}{n-p}
$$

(see (1.6)). In a sense, (1.10) can be considered as a main part of (1.11). We emphasize that the definition of $V_{k}$ contains no smoothness condition on $f$.

In the case $\alpha_{k}<1 \quad(k=1, \ldots, n)$ Theorem 1.4 closely relates to embeddings of Besov spaces.

We shall consider Besov spaces $B_{p ; k}^{\alpha}\left(\mathbb{R}^{n}\right)$ with respect to separate variables $x_{k}$ and anisotropic Besov spaces

$$
B_{p}^{\alpha_{1}, \ldots, \alpha_{k}}\left(\mathbb{R}^{n}\right)=\bigcap_{k=1}^{n} B_{p ; k}^{\alpha_{k}}\left(\mathbb{R}^{n}\right) .
$$

The definitions are given in Sections 2 and 4 below. In the isotropic case $\alpha_{1}=\cdots=\alpha_{k}=$ $\alpha$ we set $B_{p}^{\alpha}\left(\mathbb{R}^{n}\right) \equiv B_{p}^{\alpha, \ldots, \alpha}\left(\mathbb{R}^{n}\right)$.

The following theorem is well known (see [21], [7]).

Theorem 1.5. Let $0<\alpha_{k}<1(k=1, \ldots, n), \alpha=n\left(\sum_{k=1}^{n} 1 / \alpha_{k}\right)^{-1}, 1 \leq p<n / \alpha$, and $q^{*}=n p /(n-\alpha p)$. Then for every function $f \in B_{p}^{\alpha_{1}, \ldots, \alpha_{n}}\left(\mathbb{R}^{n}\right)$ we have

$$
\|f\|_{q^{*}, p} \leq c \sum_{k=1}^{n}\|f\|_{b_{p ; k}^{\alpha}} .
$$

Suppose that $\alpha_{1}=\cdots=\alpha_{n}=\alpha$. Then it follows from (1.12) that

$$
\|f\|_{q^{*}} \leq c\|f\|_{b_{p}^{\alpha}} .
$$

We have $\|f\|_{b_{p}^{\alpha}} \rightarrow \infty$ as $\alpha \rightarrow 1$, whenever $f \not 0$. Bourgain, Brezis and Mironescu [3] (see also [5]) proved that there exists a limiting relation between Sobolev and Besov norms, that is, for any $f \in W_{p}^{1}\left(\mathbb{R}^{n}\right)$,

$$
\lim _{\alpha \rightarrow 1-0}(1-\alpha)\|f\|_{b_{p}^{\alpha}}^{p}=\frac{1}{p}\|\nabla f\|_{L^{p}}^{p} .
$$


Later on Bourgain, Brezis and Mironescu [4] found the sharp asymptotic of the best constant as $\alpha \rightarrow 1$ in the inequality (1.13); namely, they proved that if $1 / 2 \leq \alpha<1$ and $1 \leq p<n / \alpha$, then for any $f \in B_{p}^{\alpha}\left(\mathbb{R}^{n}\right)$,

$$
\|f\|_{L^{q^{*}}}^{p} \leq c_{n} \frac{1-\alpha}{(n-\alpha p)^{p-1}}\|f\|_{b_{p}^{\alpha}}^{p} \quad\left(q^{*}=\frac{n p}{n-\alpha p}\right)
$$

where a constant $c_{n}$ depends only on $n$. In view of (1.14), the classical Sobolev inequality (1.1) can be considered as a limiting case of (1.15). Note that the proof given in [4] was quite complicated. Afterwards a simpler proof of this result was given in [19]. It was observed in [15] that (1.15) can be immediately derived from the rearrangement estimates obtained in [11].

In this paper we apply Theorem 1.4 to study the behaviour of the constant in (1.12) as some of the numbers $\alpha_{k}$ tend to 1 .

It is easy to prove that if $f \in B_{p ; k}^{\alpha}\left(\mathbb{R}^{n}\right) \quad(0<\alpha<1, \quad 1 \leq p<\infty)$, then $f \in V_{k} \equiv$ $L_{\hat{x}_{k}}^{p}\left[\Lambda_{x_{k}}^{1 / p-\alpha}\right]$ and

$$
\|f\|_{V_{k}} \leq 100[\alpha(1-\alpha)]^{1 / p}\|f\|_{b_{p ; k}^{\alpha}} .
$$

Using Theorem 1.4 and inequality (1.16), we obtain the following result.

Theorem 1.6. Let $1 \leq p<\infty, n \geq 2(n \in \mathbb{N})$, and $1 / 2<\alpha_{k}<1(k=1, \ldots, n)$. Assume that

$$
\alpha \equiv n\left(\sum_{k=1}^{n} \frac{1}{\alpha_{k}}\right)^{-1}<\frac{n}{p} .
$$

Let $q^{*}=n p /(n-\alpha p)$. Then for every function $f \in B_{p}^{\alpha_{1}, \ldots, \alpha_{k}}\left(\mathbb{R}^{n}\right)$ we have that $f \in$ $L^{q^{*}, p}\left(\mathbb{R}^{n}\right)$ and

$$
\|f\|_{q^{*}, p} \leq q^{*} c_{n} \prod_{k=1}^{n}\left[\left(1-\alpha_{k}\right)^{1 / p}\|f\|_{b_{p ; k}^{\alpha_{k}}}\right]^{\alpha /\left(n \alpha_{k}\right)},
$$

where $c_{n}$ is a constant depending only on $n$.

Observe that (1.10) can be considered as the main part of the embedding

$$
B_{p}^{\alpha_{1}, \ldots, \alpha_{k}} \subset L^{q^{*}, p} .
$$

The factors $\left(1-\alpha_{k}\right)^{\alpha /\left(p n \alpha_{k}\right)}$ appear due to inequality (1.16) in the "easy" part of (1.18).

We stress again that a function $f \in V_{k}$ may have bad smoothness properties (in contrast with functions in Besov or Sobolev spaces).

By the known relation between Lebesgue and Lorentz norms, (1.17) yields the inequality

$$
\|f\|_{q^{*}} \leq\left(q^{*}\right)^{1-1 / p} c_{n} \prod_{k=1}^{n}\left[\left(1-\alpha_{k}\right)^{1 / p}\|f\|_{b_{p ; k}^{\alpha_{k}}}\right]^{\alpha /\left(n \alpha_{k}\right)} .
$$

In turn, (1.19) implies (1.15).

Inequalities (1.15) and (1.19) are involved in the following general problem: given a function $f \in L^{p}\left(\mathbb{R}^{n}\right)$, find sharp estimates of its $L^{q}$-norm $(p<q<\infty)$ in terms of partial moduli of continuity of $f$. This problem was posed by Ul'yanov [24]. Ul'yanov [24] solved it for $n=1$. The complete solution in $n$-dimensional case was given in [10]. Estimates obtained in [10] are sharp in a stronger sense than the estimate (1.19) which 
is sharp only in the setting of Besov spaces. In particular, the general phenomenon of "big smoothness" found in [10] has a more complicated form than one given by (1.19) for the special case of Besov norms. The main result of [10] (Theorem 1) immediately implies (1.19). However, the proof in [10] (concerning the anisotropic case) is rather long and complicated. In the isotropic case the problem is much simpler (see [11]).

\section{Auxiliary propositions}

2.1. Moduli of continuity. For any $f \in L^{p}\left(\mathbb{R}^{n}\right), 1 \leq p<\infty$, and $h \in \mathbb{R}^{n}$, set

$$
I_{p}(h)=\left(\int_{\mathbb{R}^{n}}|f(x+h)-f(x)|^{p} d x\right)^{1 / p} .
$$

The modulus of continuity ${ }^{2}$ of a function $f$ is defined by

$$
\omega(f ; \delta)_{p}=\sup _{|h| \leq \delta} I_{p}(h) \quad(0<\delta<\infty) .
$$

Observe that $\omega(f ; \delta)_{p}$ is a non-decreasing and subadditive function. In particular, this implies that

$$
\omega(f ; \eta)_{p} / \eta \leq 2 \omega(f ; \delta)_{p} / \delta, \quad 0<\delta<\eta .
$$

Let $0<\alpha<1$ and $1 \leq p, \theta \leq \infty$. The Besov space $B_{p, \theta}^{\alpha}\left(\mathbb{R}^{n}\right)$ consists of all functions $f \in L^{p}\left(\mathbb{R}^{n}\right)$ such that

$$
\|f\|_{b_{p, \theta}^{\alpha}} \equiv\left(\int_{0}^{\infty}\left(t^{-\alpha} \omega(f ; t)_{p}\right)^{\theta} \frac{d t}{t}\right)^{1 / \theta}<\infty
$$

if $\theta<\infty$, and

$$
\|f\|_{b_{p, \infty}^{\alpha}} \equiv \sup _{t>0} t^{-\alpha} \omega(f ; t)_{p}<\infty
$$

if $\theta=\infty$. Set also $B_{p}^{\alpha} \equiv B_{p, p}^{\alpha}$.

The following lemma is well known; we outline the proof in order to get the explicit values of the constants.

Lemma 2.1. Let $f \in L^{p}(\mathbb{R}), 1 \leq p<\infty$. Then

$$
\omega(f ; \delta)_{p} \leq \frac{3}{\delta} \int_{0}^{\delta} I_{p}(h) d h, \quad \delta>0,
$$

and for any $0<\alpha<1$ and any $1 \leq \theta<\infty$

$$
\|f\|_{b_{p, \theta}^{\alpha}} \leq 3\left(\int_{0}^{\infty}\left(h^{-\alpha} I_{p}(h)\right)^{\theta} \frac{d h}{h}\right)^{1 / \theta} .
$$

Proof. Let $\delta>0$ and $0 \leq t \leq \delta$. For any $h \in[0, \delta]$ we have

$$
I_{p}(t) \leq I_{p}(t-h)+I_{p}(h) .
$$

Integrating with respect to $h$ in $[0, \delta]$, and taking into account that the function $I_{p}(u)$ is even, we get

$$
\delta I_{p}(t) \leq 3 \int_{0}^{\delta} I_{p}(h) d h,
$$

\footnotetext{
${ }^{2}$ This modulus of continuity can be called isotropic. In Section 4 we consider also partial moduli of continuity.
} 
which yields (2.2). Now, using (2.2), Hölder's inequality, and Fubini's theorem, we have

$$
\int_{0}^{\infty}\left(t^{-\alpha} \omega(f ; t)_{p}\right)^{\theta} \frac{d t}{t} \leq 3^{\theta} \int_{0}^{\infty} t^{-\alpha \theta-2} \int_{0}^{t} I_{p}(h)^{\theta} d h d t=\frac{3^{\theta}}{\alpha \theta+1} \int_{0}^{\infty} h^{-\alpha \theta} I_{p}(h)^{\theta} \frac{d h}{h} .
$$

This implies (2.3).

It is well known that

$$
B_{p, \theta}^{\alpha} \subset B_{p, \eta}^{\alpha} \quad \text { if } \quad 1 \leq \theta<\eta \leq \infty .
$$

Moreover, the following estimate holds.

LEMma 2.2. Let $1 \leq p<\infty, 1 \leq \theta<\eta \leq \infty$, and $0<\alpha<1$. Then for any function $f \in L^{p}\left(\mathbb{R}^{n}\right)$

$$
\|f\|_{b_{p, \eta}^{\alpha}} \leq 8[\alpha(1-\alpha)]^{1 / \theta-1 / \eta}\|f\|_{b_{p, \theta}^{\alpha}} .
$$

Proof. Denote $\omega(t)=\omega(f ; t)_{p}$. First, we have for any $t>0$

$$
\int_{t}^{\infty} u^{-\alpha \theta} \omega(u)^{\theta} \frac{d u}{u} \geq \omega(t)^{\theta} \int_{t}^{\infty} u^{-\alpha \theta-1} d u=\frac{1}{\alpha \theta}\left[t^{-\alpha} \omega(t)\right]^{\theta} .
$$

Next, using (2.1), we get

$$
\int_{0}^{t} u^{-\alpha \theta} \omega(u)^{\theta} \frac{d u}{u} \geq\left(\frac{\omega(t)}{2 t}\right)^{\theta} \int_{0}^{t} u^{(1-\alpha) \theta-1} d u=\frac{1}{2^{\theta}(1-\alpha) \theta}\left[t^{-\alpha} \omega(t)\right]^{\theta} .
$$

Thus, for any $t>0$

$$
t^{-\alpha} \omega(t) \leq\left[\theta \min \left(\alpha, 2^{\theta}(1-\alpha)\right)\right]^{1 / \theta}\|f\|_{b_{p, \theta}^{\alpha}} \leq\left[\left(2^{\theta}+1\right) \theta \alpha(1-\alpha)\right]^{1 / \theta}\|f\|_{b_{p, \theta}^{\alpha}} .
$$

From here we get (2.4) for $\eta=\infty$. If $\eta<\infty$, then

$$
\int_{0}^{\infty}\left[t^{-\alpha} \omega(t)\right]^{\eta} \frac{d t}{t} \leq 8^{\eta-\theta}[\alpha(1-\alpha)]^{(\eta-\theta) / \theta}\|f\|_{b_{p, \theta}^{\alpha}}^{\eta-\theta} \int_{0}^{\infty}\left[t^{-\alpha} \omega(t)\right]^{\theta} \frac{d t}{t}
$$

which again implies (2.4).

2.2. Rearrangements. Denote by $S_{0}\left(\mathbb{R}^{n}\right)$ the class of all measurable and almost everywhere finite functions $f$ on $\mathbb{R}^{n}$ such that for each $y>0$,

$$
\lambda_{f}(y) \equiv\left|\left\{x \in \mathbb{R}^{n}:|f(x)|>y\right\}\right|<\infty .
$$

A non-increasing rearrangement of a function $f \in S_{0}\left(\mathbb{R}^{n}\right)$ is a non-increasing function $f^{*}$ on $\mathbb{R}_{+} \equiv(0,+\infty)$ that is equimeasurable with $|f|$. The rearrangement $f^{*}$ can be defined by the equality

$$
f^{*}(t)=\sup _{|E|=t} \inf _{x \in E}|f(x)|
$$

The following relation holds [23, Ch. 5]:

$$
\sup _{|E|=t} \int_{E}|f(x)| d x=\int_{0}^{t} f^{*}(u) d u \text {. }
$$

In what follows we denote

$$
f^{* *}(t)=\frac{1}{t} \int_{0}^{t} f^{*}(u) d u
$$


Assume that $0<q, p<\infty$. A function $f \in S_{0}\left(\mathbb{R}^{n}\right)$ belongs to the Lorentz space $L^{q, p}\left(\mathbb{R}^{n}\right)$ if

$$
\|f\|_{q, p} \equiv\left(\int_{0}^{\infty}\left(t^{1 / q} f^{*}(t)\right)^{p} \frac{d t}{t}\right)^{1 / p}<\infty .
$$

For $0<q<\infty$, the space $L^{q, \infty}\left(\mathbb{R}^{n}\right)$ is defined as the class of all $f \in S_{0}\left(\mathbb{R}^{n}\right)$ such that

$$
\|f\|_{q, \infty} \equiv \sup _{t>0} t^{1 / q} f^{*}(t)<\infty .
$$

We have the inequality [23, p. 192]

$$
\|f\|_{q, s} \leq\left(\frac{q}{s}\right)^{1 / s}\left(\frac{p}{q}\right)^{1 / p}\|f\|_{q, p} \quad(0<p<s \leq \infty) .
$$

The space $W\left(\mathbb{R}^{n}\right) \equiv$ weak- $L^{\infty}\left(\mathbb{R}^{n}\right)$ consists of all functions $f \in S_{0}\left(\mathbb{R}^{n}\right)$ such that

$$
\|f\|_{W} \equiv \sup _{t>0}\left[f^{* *}(t)-f^{*}(t)\right]<\infty .
$$

This space was introduced by Bennett, DeVore and Sharpley [2]. It was proved in [2] that $\mathrm{BMO} \subset W$.

For a function $f \in S_{0}\left(\mathbb{R}^{n}\right)$ we consider also the quantity

$$
\omega^{*}(t) \equiv f^{*}(t)-f^{*}(2 t) .
$$

Observe that for any $t>0$

$$
\frac{1}{2} \omega^{*}\left(\frac{t}{2}\right) \leq f^{* *}(t)-f^{*}(t) \leq \frac{2}{t} \int_{0}^{t} \omega^{*}(u) d u
$$

The left hand side inequality is immediate since

$$
f^{* *}(t)-f^{*}(t) \geq \frac{1}{t} \int_{0}^{t / 2}\left[f^{*}(u)-f^{*}(t)\right] d t \geq \frac{1}{2} \omega^{*}\left(\frac{t}{2}\right) .
$$

Next, for any $t>0$ and any $0<\varepsilon<t$

$$
\int_{\varepsilon}^{t} \omega^{*}(u) d u=\int_{\varepsilon}^{t} f^{*}(u) d u-\frac{1}{2} \int_{2 \varepsilon}^{2 t} f^{*}(u) d u \geq \frac{1}{2}\left[\int_{\varepsilon}^{t} f^{*}(u) d u-t f^{*}(t)\right] .
$$

This implies the right hand side inequality in (2.8).

Similarly, we have that for any $f \in S_{0}\left(\mathbb{R}^{n}\right)$ and any $t>0$

$$
f^{*}(2 t) \leq \frac{1}{\ln 2} \int_{t}^{\infty} \frac{f^{*}(u)-f^{*}(2 u)}{u} d u .
$$

Let $1 \leq q, p<\infty$. For a function $f \in S_{0}\left(\mathbb{R}^{n}\right)$, set

$$
\|f\|_{q, p}^{*}=\left(\int_{0}^{\infty}\left[t^{1 / q}\left(f^{*}(t)-f^{*}(2 t)\right)\right]^{p} \frac{d t}{t}\right)^{1 / p}
$$

and

$$
\|f\|_{q, \infty}^{*}=\sup _{t>0} t^{1 / q}\left[f^{*}(t)-f^{*}(2 t)\right] .
$$

It follows from (2.9) and Hardy's inequality [23, p. 196] that

$$
\|f\|_{q, p} \leq \frac{2^{1 / q} q}{\ln 2}\|f\|_{q, p}^{*} \quad(1 \leq q<\infty, 1 \leq p \leq \infty) .
$$


For any $1 \leq p<\infty$ the space $L^{\infty, p}\left(\mathbb{R}^{n}\right)$ is defined as the class of all functions $f \in S_{0}\left(\mathbb{R}^{n}\right)$ such that

$$
\|f\|_{\infty, p} \equiv\left(\int_{0}^{\infty}\left[f^{* *}(t)-f^{*}(t)\right]^{p} \frac{d t}{t}\right)^{1 / p}<\infty
$$

(see [1], [18]). Set also

$$
\|f\|_{\infty, p}^{*} \equiv\left(\int_{0}^{\infty}\left[f^{*}(t)-f^{*}(2 t)\right]^{p} \frac{d t}{t}\right)^{1 / p} .
$$

It follows from (2.8) that

$$
\frac{1}{2}\|f\|_{\infty, p}^{*} \leq\|f\|_{\infty, p} \leq 2\|f\|_{\infty, p}^{*} .
$$

Recall that the space $\Lambda^{\sigma}(\mathbb{R}) \quad(\sigma \in \mathbb{R})$ is defined as the class of all functions $f \in S_{0}(\mathbb{R})$ such that

$$
\|f\|_{\Lambda^{\sigma}} \equiv \sup _{t>0} t^{\sigma}\left[f^{*}(t)-f^{*}(2 t)\right]<\infty .
$$

If $0<\sigma<\infty$ and $r=1 / \sigma$, then by $(2.9)$ for any $f \in S_{0}(\mathbb{R})$

$$
\|f\|_{\Lambda^{\sigma}} \leq\|f\|_{r, \infty} \leq \frac{2^{\sigma+1}}{\sigma}\|f\|_{\Lambda^{\sigma}} .
$$

Thus, $\Lambda^{\sigma}(\mathbb{R})=L^{r, \infty}(\mathbb{R})$. If $\sigma=0$, then $\Lambda^{\sigma}$ coincides with the space weak- $L^{\infty}$ (see $(2.7)$ and $(2.8))$.

The following lemma was obtained in [12, Lemma 5.1]. As it was observed in [12], this lemma follows by simple arguments contained in [11, Theorem 2]. We give these arguments here.

LEMMA 2.3. Let $f \in S_{0}\left(\mathbb{R}^{n}\right)$ be a locally integrable function which has all weak derivatives $\partial f / \partial x_{k} \in L_{l o c}^{1}(k=1, \ldots, n)$. Then

$$
f^{* *}(t)-f^{*}(t) \leq \sqrt{n} t^{1 / n}(|\nabla f|)^{* *}(t) .
$$

Proof. Let $x \in \mathbb{R}^{n}$ and $t>0$. Denote by $Q_{x}(t)$ the cube centered at $x$ with side length $(2 t)^{1 / n}$. Fix $x$ and set

$$
A_{t}=\left\{y \in Q_{x}(t):|f(y)| \leq f^{*}(t)\right\} .
$$

Then $\left|A_{t}\right| \geq t$. For any $y \in A_{t}$

$$
|f(x)|-f^{*}(t) \leq|f(x)|-|f(y)| \leq|f(x)-f(y)| .
$$

Integrating over $A_{t}$, we have

$$
|f(x)|-f^{*}(t) \leq \frac{1}{t} \int_{A_{t}}|f(x)-f(y)| d y \leq \frac{1}{t} \int_{Q_{0}(t)}|f(x)-f(x+h)| d h .
$$

For each $h \in \mathbb{R}^{n}$ and almost every $x \in \mathbb{R}^{n}$

$$
f(x+h)-f(x)=\int_{0}^{1} \nabla f(x+\tau h) \cdot h d \tau
$$

(see $\left[16\right.$, p. 135]). Thus, for almost all $x \in \mathbb{R}^{n}$

$$
|f(x)|-f^{*}(t) \leq \sqrt{n} t^{1 / n-1} \int_{0}^{1} d \tau \int_{Q_{0}(t)}|\nabla f(x+\tau h)| d h .
$$


Let $E \subset \mathbb{R}^{n},|E|=t$. Then for all $\tau \in[0,1], h \in Q_{0}(t)$

$$
\int_{E}|\nabla f(x+\tau h)| d x \leq \int_{0}^{t}(|\nabla f|)^{*}(u) d u .
$$

Applying (2.5), we get (2.14).

Observe that estimate (2.14) can be efficiently applied in the study of Sobolev spaces $W_{p}^{1}$ in the case $p>1$. In particular, inequality (1.6) for $1<p<n$ follows at once from (2.14) and Hardy's inequality. In the limiting case $p=n$ estimate (2.14) and Hardy's inequality immediately imply that for any function $f \in W_{n}^{1}\left(\mathbb{R}^{n}\right) \quad(n \geq 2)$

$$
\left(\int_{0}^{\infty}\left[f^{* *}(t)-f^{*}(t)\right]^{n} \frac{d t}{t}\right)^{1 / n} \leq c_{n}\|\nabla f\|_{n}
$$

i.e., $W_{n}^{1}\left(\mathbb{R}^{n}\right) \subset L^{\infty, n}\left(\mathbb{R}^{n}\right)$ (see [1], [18]). Observe also that $(2.15)$ can be considered as a special case of the inequality (2.23) in [11].

LEMma 2.4. Let $f \in L^{p}\left(\mathbb{R}^{n}\right), 1 \leq p<\infty$. Then for any $t>0$

$$
f^{* *}(t)-f^{*}(t) \leq 2^{1 / p} t^{-1 / p} \omega\left(f ; t^{1 / n}\right)_{p} .
$$

This estimate was first proved by Ul'yanov [24] in the one-dimensional case. A simple proof in the general case is contained in [11, Theorem 1].

\subsection{Hardy type inequality}

LEMmA 2.5. Let $f$ be a nonnegative non-increasing function on $\mathbb{R}_{+}$. Suppose that $\beta>0$ and $0<r<1$. Then

$$
\int_{0}^{\infty} t^{\beta-1}\left(\int_{t}^{\infty} f(u) d u\right)^{r} d t \leq e\left(1+\frac{r}{\beta}\right) \int_{0}^{\infty} t^{\beta+r-1} f(t)^{r} d t .
$$

Proof. We can assume that $f$ is a bounded function with compact support. Since $f$ is non-increasing, then we have for any $A>1$

$$
\begin{aligned}
J & \equiv \int_{0}^{\infty} t^{\beta-1}\left(\int_{t}^{\infty} f(u) d u\right)^{r} d t \\
& \leq \int_{0}^{\infty} t^{\beta-1}\left(\int_{t}^{A t} f(u) d u\right)^{r} d t+\int_{0}^{\infty} t^{\beta-1}\left(\int_{A t}^{\infty} f(u) d u\right)^{r} d t \\
& \leq(A-1)^{r} J^{*}+A^{-\beta} J,
\end{aligned}
$$

where

$$
J^{*}=\int_{0}^{\infty} t^{\beta+r-1} f(t)^{r} d t
$$

It follows that

$$
J \leq \frac{A^{r+\beta}}{A^{\beta}-1} J^{*} .
$$

Taking $A=(1+\beta / r)^{1 / \beta}$, we get $(2.17)$.

2.4. Projections and sections. As above, for any vector $x \in \mathbb{R}^{n}$ and every $k \in\{1, \ldots, n\}$ we denote by $\hat{x}_{k}$ the $(n-1)$-dimensional vector obtained from $x$ by removal of its $k$ th coordinate. We write also $x=\left(x_{k}, \hat{x}_{k}\right)$. 
Let $f \in S_{0}\left(\mathbb{R}^{n}\right)$ and assume that $1 \leq k \leq n$. Fix $\hat{x}_{k} \in \mathbb{R}^{n-1}$ and consider the function of the variable $x_{k}$

$$
f_{\hat{x}_{k}}\left(x_{k}\right)=f\left(x_{k}, \hat{x}_{k}\right), \quad x_{k} \in \mathbb{R} .
$$

For almost all $\hat{x}_{k} \in \mathbb{R}^{n-1}$ we have $f_{\hat{x}_{k}} \in S_{0}(\mathbb{R})$. We set

$$
\mathcal{R}_{k} f\left(t, \hat{x}_{k}\right)=\left(f_{\hat{x}_{k}}\right)^{*}(t), \quad t>0 .
$$

The function $\mathcal{R}_{k} f$ is defined almost everywhere on $\mathbb{R}_{+} \times \mathbb{R}^{n-1}$. We call it the rearrangement of $f$ with respect to $k$ th variable. It is a measurable function equimeasurable with $|f|$.

Let $1 \leq p<\infty, n \geq 2 \quad(n \in \mathbb{N})$, and $\sigma \in \mathbb{R}$. In this paper we consider the spaces

$$
V_{k} \equiv L_{\hat{x}_{k}}^{p}\left(\mathbb{R}^{n-1}\right)\left[\Lambda_{x_{k}}^{\sigma}(\mathbb{R})\right]
$$

with the norm

$$
\|f\|_{V_{k}}=\left(\int_{\mathbb{R}^{n-1}}\left[\psi_{k}\left(\hat{x}_{k}\right)\right]^{p} d \hat{x}_{k}\right)^{1 / p},
$$

where $\psi_{k}\left(\hat{x}_{k}\right)=\left\|f_{\hat{x}_{k}}\right\|_{\Lambda^{\sigma}}=\sup _{t>0} t^{\sigma}\left[\mathcal{R}_{k} f\left(t, \hat{x}_{k}\right)-\mathcal{R}_{k} f\left(2 t, \hat{x}_{k}\right)\right]$.

Let $E \subset \mathbb{R}^{n}$. For every $k=1, \ldots, n$, denote by $\Pi_{k}(E)$ the orthogonal projection of $E$ onto the coordinate hyperplane $x_{k}=0$. Further, if $\hat{x}_{k} \in \mathbb{R}^{n-1}$, then by $E\left(\hat{x}_{k}\right)$ we denote the $x_{k}$-section of $E$,

$$
E\left(\hat{x}_{k}\right)=\left\{x_{k}:\left(x_{k}, \hat{x}_{k}\right) \in E\right\} .
$$

The Lebesgue measure of a measurable set $A \subset \mathbb{R}^{k}$ will be denoted by $\operatorname{mes}_{k} A$.

The following lemma was proved by Loomis and Whitney [17] (it follows also from Lemma 1.1).

Lemma 2.6. Let $E \subset \mathbb{R}^{n}$ be a set of type $F_{\sigma}$. Then

$$
\left(\operatorname{mes}_{n} E\right)^{n-1} \leq \prod_{k=1}^{n} \operatorname{mes}_{n-1} \Pi_{k}(E) .
$$

\section{Embeddings of mixed norm spaces}

THEOREM 3.1. Assume that $1 \leq p<\infty, n \geq 2(n \in \mathbb{N})$, and that $\alpha_{k}(k=1, \ldots, n)$ are positive numbers such that

$$
\alpha \equiv n\left(\sum_{k=1}^{n} \frac{1}{\alpha_{k}}\right)^{-1} \leq \frac{n}{p} .
$$

Let

$$
\sigma_{k}=\frac{1}{p}-\alpha_{k}, \quad V_{k} \equiv L_{\hat{x}_{k}}^{p}\left(\mathbb{R}^{n-1}\right)\left[\Lambda_{x_{k}}^{\sigma_{k}}(\mathbb{R})\right]
$$

and

$$
q=\frac{n p}{n-\alpha p}
$$

Suppose that

$$
f \in S_{0}\left(\mathbb{R}^{n}\right) \quad \text { and } \quad f \in V_{p}^{\alpha_{1}, \ldots, \alpha_{n}} \equiv \bigcap_{k=1}^{n} V_{k}
$$


Then $f \in L^{q, p}\left(\mathbb{R}^{n}\right)$ and

$$
\|f\|_{q, p}^{*} \leq c \prod_{k=1}^{n}\|f\|_{V_{k}}^{\alpha /\left(n \alpha_{k}\right)}
$$

where

$$
c=c_{n}\left(\prod_{k=1}^{n}\left(n \alpha_{k}-\alpha\right)^{\alpha /\left(n \alpha_{k}\right)}\right)^{-1 / p}
$$

and $c_{n}$ is a constant depending only on $n$.

Proof. We can suppose that $f$ is a nonnegative continuous function with compact support.

Fix $t>0$ and estimate the difference $f^{*}(t)-f^{*}(2 t)$. We assume that this difference is positive. It is easy to see that there exist two bounded $F_{\sigma}$-sets in $\mathbb{R}^{n}$, a set $A$ with mes $_{n} A=t$ and a set $B$ with $\operatorname{mes}_{n} B=2 t$, such that $A \subset B$,

$$
\left\{x: f(x)>f^{*}(t)\right\} \subset A \subset\left\{x: f(x) \geq f^{*}(t)\right\},
$$

and

$$
\left\{x: f(x)>f^{*}(2 t)\right\} \subset B \subset\left\{x: f(x) \geq f^{*}(2 t)\right\} .
$$

Denote

$$
\alpha_{j}\left(\hat{x}_{j}\right)=\operatorname{mes}_{1} A\left(\hat{x}_{j}\right), \quad \beta_{j}\left(\hat{x}_{j}\right)=\operatorname{mes}_{1} B\left(\hat{x}_{j}\right) .
$$

Since $f$ is continuous, then it follows from (3.4) and (3.5) that for all $\hat{x}_{j} \in \Pi_{j}(A) \quad(j=$ $1, \ldots, n)$

$$
\mathcal{R}_{j} f\left(\alpha_{j}\left(\hat{x}_{j}\right), \hat{x}_{j}\right)=f^{*}(t) \quad \text { and } \quad \mathcal{R}_{j} f\left(\beta_{j}\left(\hat{x}_{j}\right), \hat{x}_{j}\right)=f^{*}(2 t) .
$$

For every $j=1, \ldots, n$, denote by $P_{j}$ the set of all $\hat{x}_{j} \in \Pi_{j}(A)$ such that

$$
\beta_{j}\left(\hat{x}_{j}\right) \leq 2^{n+1} \alpha_{j}\left(\hat{x}_{j}\right) .
$$

Let $A_{j}=\left\{x \in A: \hat{x}_{j} \in P_{j}\right\}$. Then

$$
\operatorname{mes}_{n} A_{j} \geq\left(1-2^{-n}\right) t, \quad j=1, \ldots, n .
$$

Indeed, if for some $j$ the opposite inequality was true, then we would have that

$$
\operatorname{mes}_{n} B \geq \int_{\Pi_{j}(A) \backslash P_{j}} \beta_{j}\left(\hat{x}_{j}\right) d \hat{x}_{j}>2^{n+1} \int_{\Pi_{j}(A) \backslash P_{j}} \alpha_{j}\left(\hat{x}_{j}\right) d \hat{x}_{j}=2^{n+1} \operatorname{mes}_{n}\left(A \backslash A_{j}\right)>2 t,
$$

which is false.

Denote

$$
\widetilde{A}=\bigcap_{j=1}^{n} A_{j}
$$

By (3.8),

$$
\operatorname{mes}_{n} \widetilde{A} \geq \frac{t}{2} .
$$

Set

$$
\gamma_{j}=1-\frac{\alpha}{n \alpha_{j}}, \quad j=1, \ldots, n
$$

Then $0<\gamma_{j}<1$ and

$$
\sum_{j=1}^{n} \gamma_{j}=n-1
$$


Let $\Omega$ be the set of all $k \in\{1, \ldots, n\}$ for which

$$
\operatorname{mes}_{n-1} P_{k} \geq \frac{t^{\gamma_{k}}}{2}
$$

Since $\Pi_{k}(\widetilde{A}) \subset P_{k}$, it follows from (3.9), (3.10), and the Loomis-Whitney inequality (2.18) that $\Omega \neq \emptyset$.

Denote

$$
\psi_{j}\left(\hat{x}_{j}\right)=\left\|f\left(\cdot, \hat{x}_{j}\right)\right\|_{\Lambda^{\sigma_{j}}}, \quad j=1, \ldots, n .
$$

Let $k \in \Omega$. First we suppose that $\sigma_{k} \leq 0$. By (3.6) and (3.7), we have for all $\hat{x}_{k} \in P_{k}$

$$
\begin{aligned}
f^{*}(t)-f^{*}(2 t) & =\mathcal{R}_{k} f\left(\alpha_{k}\left(\hat{x}_{k}\right), \hat{x}_{k}\right)-\mathcal{R}_{k} f\left(\beta_{k}\left(\hat{x}_{k}\right), \hat{x}_{k}\right) \\
& \leq(n+1) 2^{\left|\sigma_{k}\right| n}\left(\alpha_{k}\left(\hat{x}_{k}\right)\right)^{-\sigma_{k}} \psi_{k}\left(\hat{x}_{k}\right) .
\end{aligned}
$$

Let

$$
P_{k}^{\prime}=\left\{\hat{x}_{k} \in P_{k}: \alpha_{k}\left(\hat{x}_{k}\right) \leq 4 t^{1-\gamma_{k}}\right\} .
$$

Then $\operatorname{mes}_{n-1} P_{k}^{\prime} \geq t^{\gamma_{k}} / 4$. Indeed, otherwise by (3.11) we would have that

$$
\operatorname{mes}_{n} A \geq \int_{P_{k} \backslash P_{k}^{\prime}} \alpha_{k}\left(\hat{x}_{k}\right) d \hat{x}_{k}>t
$$

which is false.

Taking in (3.12) the infimum over all $\hat{x}_{k} \in P_{k}^{\prime}$, we obtain that if $k \in \Omega$ and $\sigma_{k} \leq 0$, then

$$
f^{*}(t)-f^{*}(2 t) \leq(n+1) 4^{\left|\sigma_{k}\right| n} t^{\left(\gamma_{k}-1\right) \sigma_{k}} \psi_{k}^{*}\left(t^{\gamma_{k}} / 4\right) .
$$

Now suppose that for all $k \in \Omega$ we have $\sigma_{k}>0$. Let $r_{k}=1 / \sigma_{k}$. Applying (3.6) and (3.7), we have for all $\hat{x}_{k} \in P_{k} \quad(k \in \Omega)$

$$
\alpha_{k}\left(\hat{x}_{k}\right)\left[f^{*}(t)-f^{*}(2 t)\right]^{r_{k}} \leq(n+1)^{r_{k}}\left(\psi_{k}\left(\hat{x}_{k}\right)\right)^{r_{k}} .
$$

Taking into account (3.11) and using Lemma 3 of [13], we split $P_{k}$ into two disjoint measurable sets $Q_{k}^{\prime}$ and $Q_{k}^{\prime \prime}$ such that $\operatorname{mes}_{n-1} Q_{k}^{\prime}=t^{\gamma_{k}} / 2$ and

$$
\int_{Q_{k}^{\prime \prime}}\left(\psi_{k}\left(\hat{x}_{k}\right)\right)^{r_{k}} d \hat{x}_{k} \leq \int_{t^{\gamma_{k} / 2}}^{\infty}\left(\psi_{k}^{*}(u)\right)^{r_{k}} d u
$$

We consider two cases.

First we suppose that there exists $k \in \Omega$ such that the set $A_{k}^{\prime \prime}=\left\{x \in \widetilde{A}: \hat{x}_{k} \in Q_{k}^{\prime \prime}\right\}$ has measure $\operatorname{mes}_{n} A_{k}^{\prime \prime} \geq t /(4 n)$. Then we fix such $k$ and integrate inequality (3.14) over $Q_{k}^{\prime \prime}$. Note that

$$
\int_{Q_{k}^{\prime \prime}} \alpha_{k}\left(\hat{x}_{k}\right) d \hat{x}_{k} \geq \operatorname{mes}_{n} A_{k}^{\prime \prime} \geq \frac{t}{4 n} .
$$

Thus, in virtue of (3.15), we get

$$
f^{*}(t)-f^{*}(2 t) \leq 4^{\sigma_{k}}(n+1)^{1+\sigma_{k}} t^{-\sigma_{k}}\left(\int_{t^{\gamma_{k} / 2}}^{\infty}\left(\psi_{k}^{*}(u)\right)^{r_{k}} d u\right)^{1 / r_{k}} .
$$

Now we suppose that for all $k \in \Omega$ we have $\operatorname{mes}_{n} A_{k}^{\prime \prime}<t /(4 n)$. Set $A^{\prime}=\widetilde{A} \backslash\left(\cup_{k \in \Omega} A_{k}^{\prime \prime}\right)$. Then

$$
\operatorname{mes}_{n} A^{\prime} \geq \frac{t}{4}
$$


(see (3.9)). We have also

$$
\operatorname{mes}_{n-1} \Pi_{j}\left(A^{\prime}\right) \leq \frac{t^{\gamma_{j}}}{2} \quad(j=1, \ldots, n) .
$$

Indeed, $\Pi_{j}\left(A^{\prime}\right) \subset \Pi_{j}(\widetilde{A}) \subset P_{j}$ for any $j=1, \ldots, n$. By the definition of $\Omega$ (see $(3.11)$,

$$
\operatorname{mes}_{n-1} P_{j} \leq \frac{t^{\gamma_{j}}}{2} \quad \text { for all } j \notin \Omega \text {. }
$$

Thus, (3.18) is true for all $j \notin \Omega$. Let now $j \in \Omega$. Then

$$
\Pi_{j}\left(A^{\prime}\right) \subset P_{j} \backslash Q_{j}^{\prime \prime}=Q_{j}^{\prime} ;
$$

but $\operatorname{mes}_{n-1} Q_{j}^{\prime}=t^{\gamma_{j}} / 2$. Therefore (3.18) holds also for all $j \in \Omega$.

Fix $k \in \Omega$. Let $S$ be the set of all $x=\left(x_{k}, \hat{x}_{k}\right) \in A^{\prime}$ for which

$$
\operatorname{mes}_{1} A^{\prime}\left(\hat{x}_{k}\right) \geq \frac{t^{1-\gamma_{k}}}{4} \text {. }
$$

By (3.17) and (3.18) we have

$$
\operatorname{mes}_{n} S \geq \frac{t}{8}
$$

Furthermore, by (3.18),

$$
\operatorname{mes}_{n-1} \Pi_{j}(S) \leq \frac{t^{\gamma_{j}}}{2} \quad(j=1, \ldots, n) .
$$

It follows from (3.20), (3.21), and the Loomis-Whitney inequality (2.18) that

$$
\operatorname{mes}_{n-1} \Pi_{k}(S) \geq \frac{t^{\gamma_{k}}}{4^{n-1}} .
$$

For all $\hat{x}_{k} \in \Pi_{k}(S)$ we have by $(3.19)$

$$
\alpha_{k}\left(\hat{x}_{k}\right) \geq \frac{t^{1-\gamma_{k}}}{4}
$$

Thus, for every $\hat{x}_{k} \in \Pi_{k}(S)$ we obtain from (3.14) and (3.22)

$$
f^{*}(t)-f^{*}(2 t) \leq 4^{\sigma_{k}}(n+1) t^{\left(\gamma_{k}-1\right) \sigma_{k}} \psi_{k}^{*}\left(4^{1-n} t^{\gamma_{k}}\right) .
$$

Note that

$$
\psi_{k}^{*}\left(4^{1-n} t^{\gamma_{k}}\right) \leq 4^{n \sigma_{k}} t^{-\gamma_{k} \sigma_{k}}\left(\int_{4^{-n} t^{\gamma_{k}}}^{\infty}\left(\psi_{k}^{*}(u)\right)^{1 / \sigma_{k}} d u\right)^{\sigma_{k}} .
$$

Thus, it follows from (3.16) and (3.23) that if $\sigma_{k}>0$ for all $k \in \Omega$, then there exists $k \in \Omega$ such that

$$
f^{*}(t)-f^{*}(2 t) \leq 4^{(n+1) \sigma_{k}}(n+1) t^{-\sigma_{k}}\left(\int_{4^{-n} t^{\gamma_{k}}}^{\infty}\left(\psi_{k}^{*}(u)\right)^{1 / \sigma_{k}} d u\right)^{\sigma_{k}} .
$$

Combining this with (3.13), we obtain that

$$
\begin{aligned}
\int_{0}^{\infty}\left[t^{1 / q}\left(f^{*}(t)-f^{*}(2 t)\right)\right]^{p} \frac{d t}{t} & \leq(n+1)^{p}\left(\sum_{\sigma_{k} \leq 0} 4^{n p\left|\sigma_{k}\right|} \int_{0}^{\infty} t^{p\left(1 / q+\left(\gamma_{k}-1\right) \sigma_{k}\right)}\left(\psi_{k}^{*}\left(t^{\gamma_{k}} / 4\right)\right)^{p} \frac{d t}{t}\right. \\
& \left.+4^{n+1} \sum_{\sigma_{k}>0} \int_{0}^{\infty} t^{p\left(1 / q-\sigma_{k}\right)}\left(\int_{4^{-n} t^{\gamma_{k}}}^{\infty}\left(\psi_{k}^{*}(u)\right)^{1 / \sigma_{k}} d u\right)^{p \sigma_{k}} \frac{d t}{t}\right) .
\end{aligned}
$$


Let $\sigma_{k} \leq 0$. Since $1 / q+\left(\gamma_{k}-1\right) \sigma_{k}=\gamma_{k} / p$, we have

$$
\int_{0}^{\infty} t^{p\left(1 / q+\left(\gamma_{k}-1\right) \sigma_{k}\right)}\left(\psi_{k}^{*}\left(t^{\gamma_{k}} / 4\right)\right)^{p} \frac{d t}{t}=\frac{4}{\gamma_{k}} \int_{0}^{\infty}\left(\psi_{k}^{*}(t)\right)^{p} d t=\frac{4}{\gamma_{k}}\|f\|_{V_{k}}^{p} .
$$

Next, suppose that $\sigma_{k}>0$ for some $k$. Applying Lemma 2.5 with $r=p \sigma_{k}$ and $\beta=p \alpha_{k}$, we obtain

$$
\begin{aligned}
\int_{0}^{\infty} t^{p\left(1 / q-\sigma_{k}\right)}\left(\int_{4^{-n} t^{\gamma_{k}}}^{\infty}\left(\psi_{k}^{*}(u)\right)^{1 / \sigma_{k}}\right. & d u)^{p \sigma_{k}} \frac{d t}{t} \\
& =\frac{4^{n p \alpha_{k}}}{\gamma_{k}} \int_{0}^{\infty} t^{p \alpha_{k}}\left(\int_{t}^{\infty}\left(\psi_{k}^{*}(u)\right)^{1 / \sigma_{k}} d u\right)^{p \sigma_{k}} \frac{d t}{t} \\
& \leq \frac{4^{n p \alpha_{k}+1}}{\alpha_{k} \gamma_{k}} \int_{0}^{\infty}\left(\psi_{k}^{*}(t)\right)^{p} d t=\frac{4^{n p \alpha_{k}+1}}{\alpha_{k} \gamma_{k}}\|f\|_{V_{k}}^{p} .
\end{aligned}
$$

From these estimates we get that

$$
\begin{aligned}
\|f\|_{q, p}^{*} & \equiv\left(\int_{0}^{\infty}\left[t^{1 / q}\left(f^{*}(t)-f^{*}(2 t)\right)\right]^{p} \frac{d t}{t}\right)^{1 / p} \\
& \leq 2^{5 n} \sum_{k=1}^{n} \mu_{k}\|f\|_{V_{k}}, \quad \text { where } \mu_{k}=4^{(n+1) \alpha_{k}}\left(\alpha_{k} \gamma_{k}\right)^{-1 / p} .
\end{aligned}
$$

It remains to show that (3.24) can be transformed into multiplicative inequality (3.2). We apply standard arguments. Set

$$
\varepsilon_{k}=\left(\mu_{k}\|f\|_{V_{k}}\right)^{-1 / \alpha_{k}}, \quad \varepsilon=\prod_{k=1}^{n} \varepsilon_{k},
$$

and $g(x)=f\left(\varepsilon_{1} x_{1}, \ldots, \varepsilon_{n} x_{n}\right)$. It is easy to see that $g^{*}(t)=f^{*}(\varepsilon t), \quad\|g\|_{q, p}^{*}=\varepsilon^{-1 / q}\|f\|_{q, p}^{*}$, and

$$
\|g\|_{V_{k}}=\varepsilon_{k}^{-\sigma_{k}}\left(\prod_{j \neq k} \varepsilon_{j}\right)^{-1 / p}\|f\|_{V_{k}}
$$

Using these relations, applying (3.24) to the function $g$, and taking into account (3.1), we easily get that

$$
\|f\|_{q, p}^{*} \leq c_{n} \varepsilon^{-\alpha / n} \leq c_{n}^{\prime} \prod_{k=1}^{n}\left[\left(\alpha_{k} \gamma_{k}\right)^{-1 / p}\|f\|_{V_{k}}\right]^{\alpha /\left(n \alpha_{k}\right)} .
$$

This implies (3.2).

Since $f \in S_{0}\left(\mathbb{R}^{n}\right)$, then by $(3.2),(2.12)$, and $(2.13)$ we have that $f \in L^{q, p}$.

REMARK 3.2. If at least one of the numbers $\alpha_{k}$ tends to 0 , then the constant $c$ in (3.2) tends to infinity. The order of growth of this constant given by (3.3) is optimal. To show this, consider the following example (for $n=2$ ). Let $1 \leq p<\infty, \quad 0<\alpha_{1}, \alpha_{2}<1 / p$,

$$
\alpha=2\left(\frac{1}{\alpha_{1}}+\frac{1}{\alpha_{2}}\right)^{-1}, \text { and } q=\frac{2 p}{2-\alpha p} .
$$

Let $f(x, y)=x^{\alpha_{1}-1 / p} y^{\alpha_{2}-1 / p}$ if $(x, y) \in(0,1)^{2}$ and $f(x, y)=0$ otherwise. Then

$$
\|f\|_{V_{1}}=\left(p \alpha_{2}\right)^{-1 / p}, \quad\|f\|_{V_{2}}=\left(p \alpha_{1}\right)^{-1 / p},
$$


and

$$
\|f\|_{q}=\left(\frac{2}{q}\right)^{2 / q}\left[\left(2 \alpha_{1}-\alpha\right)\left(2 \alpha_{2}-\alpha\right)\right]^{-1 / q} .
$$

Denote (see (3.3))

$$
A=\left[\left(2 \alpha_{1}-\alpha\right)^{\alpha /\left(2 \alpha_{1}\right)}\left(2 \alpha_{2}-\alpha\right)^{\alpha /\left(2 \alpha_{2}\right)}\right]^{-1 / p} .
$$

For all $x>0$ we have $x^{x}>1 / 2$. Thus,

$$
\begin{aligned}
\|f\|_{q} & =\left(\frac{2}{q}\right)^{2 / q} A\left[\left(2 \alpha_{1}-\alpha\right)^{1-1 /\left(p \alpha_{2}\right)}\left(2 \alpha_{2}-\alpha\right)^{1-1 /\left(p \alpha_{1}\right)}\right]^{\alpha / 2} \\
& =\left(\frac{2}{q}\right)^{2 / q} A \alpha^{\alpha-1 / p}\left[\alpha_{1}^{1 / \alpha_{1}-1 / \alpha_{2}} \alpha_{2}^{1 / \alpha_{2}-1 / \alpha_{1}}\right]^{\alpha /(2 p)} \\
& \geq \frac{p^{1 / p}}{4} A\|f\|_{V_{1}}^{\alpha /\left(2 \alpha_{1}\right)}\|f\|_{V_{2}}^{\alpha /\left(2 \alpha_{2}\right)}\left(\frac{\alpha_{1}^{\alpha /\left(2 \alpha_{1}\right)} \alpha_{2}^{\alpha /\left(2 \alpha_{2}\right)}}{\alpha}\right)^{1 / p} .
\end{aligned}
$$

Next, $\alpha / 2 \leq \min \left(\alpha_{1}, \alpha_{2}\right)$. Therefore

$$
\|f\|_{q} \geq \frac{A}{8}\|f\|_{V_{1}}^{\alpha /\left(2 \alpha_{1}\right)}\|f\|_{V_{2}}^{\alpha /\left(2 \alpha_{2}\right)} .
$$

By (2.12) and (2.6),

$$
\|f\|_{q, p}^{*} \geq \frac{\ln 2}{q}\|f\|_{q, p} \geq \frac{\ln 2}{q}\left(\frac{q}{p}\right)^{1 / p}\|f\|_{q} .
$$

These estimates yield that the constant in (3.2) is optimal.

Observe that in the case $\alpha_{1}=\cdots=\alpha_{n}=\alpha$ the constant (3.3) equals to $c_{n} \alpha^{-1 / p}$.

REMARK 3.3. Suppose that $\alpha_{k}=1, k=1, \ldots, n$.

If $p=1$, then $\sigma_{k}=0 \quad(k=1, \ldots, n), q=n /(n-1)$, and

$$
V_{k}=L_{\hat{x}_{k}}^{1}\left[\text { weak- } L_{x_{k}}^{\infty}\right] \text {. }
$$

We have from Theorem 3.1

$$
\|f\|_{n /(n-1), 1} \leq c\left(\prod_{k=1}^{n}\|f\|_{V_{k}} B\right)^{1 / n} .
$$

This inequality is slightly stronger than the Fournier inequality (1.7). Indeed, the right hand side of (1.7) contains the norms in the spaces $L_{\hat{x}_{k}}^{1}\left[L_{x_{k}}^{\infty}\right]$. We have proved that the interior $L_{x_{k}}^{\infty}$-norms can be replaced by weaker norms of weak- $L_{x_{k}}^{\infty}$.

If $1<p \leq n$, then $\sigma_{k}=1 / p-1 \quad(k=1, \ldots, n)$ and $V_{k}=L_{\hat{x}_{k}}^{p}\left[\Lambda_{x_{k}}^{1 / p-1}\right]$. In this case Theorem 3.1 asserts that

$$
\|f\|_{q, p} \leq c\left(\prod_{k=1}^{n}\|f\|_{V_{k}}\right)^{1 / n}, \quad \text { where } \quad q=\frac{n p}{n-p} .
$$

If $p=n$, then $q=\infty$ and we have the norm in $L^{\infty, n}\left(\mathbb{R}^{n}\right)$ at the left hand side.

It is easy to see that these results are closely connected with Sobolev type inequalities (1.6) and (2.15). 
Proposition 3.4. Let $k \in\{1, \ldots, n\}$ and $1 \leq p<\infty$. Assume that $f \in L^{p}\left(\mathbb{R}^{n}\right)$ and that $f$ has the weak partial derivative $D_{k} f \in L^{p}\left(\mathbb{R}^{n}\right)$. Then $f \in V_{k} \equiv L_{\hat{x}_{k}}^{p}\left[\Lambda_{x_{k}}^{1 / p-1}\right]$ and

$$
\|f\|_{V_{k}} \leq 4\left\|D_{k} f\right\|_{p}
$$

Proof. Indeed, by (2.14) and (2.8) we have for almost all $\hat{x}_{k} \in \mathbb{R}^{n-1}$

$$
\mathcal{R}_{k} f\left(t, \hat{x}_{k}\right)-\mathcal{R}_{k} f\left(2 t, \hat{x}_{k}\right) \leq 4 \int_{0}^{t}\left(D_{k} f\right)^{*}(u) d u \leq 4 t^{1-1 / p}\left(\int_{\mathbb{R}}\left|D_{k} f(x)\right|^{p} d x_{k}\right)^{1 / p} .
$$

Thus, for almost all $\hat{x}_{k}$

$$
\left\|f\left(\cdot, \hat{x}_{k}\right)\right\|_{\Lambda_{x_{k}}^{1 / p-1}} \leq 4\left(\int_{\mathbb{R}}\left|D_{k} f(x)\right|^{p} d x_{k}\right)^{1 / p} .
$$

This implies (3.25).

Recall that

$$
W_{p}^{1}\left(\mathbb{R}^{n}\right) \subset L^{q, p}\left(\mathbb{R}^{n}\right), \quad 1 \leq p \leq n, q=\frac{n p}{n-p} .
$$

(see (1.6) and (2.15)). At the same time, by Theorem 3.1

$$
\bigcap_{k=1}^{n} V_{k} \subset L^{q, p}\left(\mathbb{R}^{n}\right)
$$

and by Proposition 3.4,

$$
W_{p}^{1}\left(\mathbb{R}^{n}\right) \subset \bigcap_{k=1}^{n} V_{k} .
$$

Thus, we can split (3.26) into two embeddings (3.27) and (3.28). Clearly, (3.27) is the main part of (3.26).

4. Limiting embeddings. Let $f \in L^{p}\left(\mathbb{R}^{n}\right)$ and $k \in\{1, \ldots, n\}$. The partial modulus of continuity of $f$ in $L^{p}$ with respect to $x_{k}$ is defined by

$$
\omega_{k}(f ; \delta)_{p}=\sup _{0 \leq h \leq \delta}\left(\int_{\mathbb{R}^{n}}\left|f\left(x+h e_{k}\right)-f(x)\right|^{p} d x\right)^{1 / p} .
$$

( $e_{k}$ is the $k$ th unit coordinate vector). It is easy to see that

$$
\max _{k} \omega_{k}(f ; \delta)_{p} \leq \omega(f ; \delta)_{p} \leq \sum_{k=1}^{n} \omega_{k}(f ; \delta)_{p} .
$$

Let $0<\alpha<1, k \in\{1, \ldots, n\}$, and $1 \leq p, \theta<\infty$. The Besov space $B_{p, \theta ; k}^{\alpha}\left(\mathbb{R}^{n}\right)$ is defined as the space of all functions $f \in L^{p}\left(\mathbb{R}^{n}\right)$ such that

$$
\|f\|_{b_{p, \theta ; k}^{\alpha}} \equiv\left(\int_{0}^{\infty}\left(t^{-\alpha} \omega_{k}(f ; t)_{p}\right)^{\theta} \frac{d t}{t}\right)^{1 / \theta}<\infty .
$$

Denote also $B_{p, p ; k}^{\alpha} \equiv B_{p ; k}^{\alpha}$.

Let $0<\alpha_{k}<1 \quad(k=1, \ldots, n)$ and $1 \leq p, \theta<\infty$. Then we set

$$
B_{p, \theta}^{\alpha_{1}, \ldots, \alpha_{k}}\left(\mathbb{R}^{n}\right)=\bigcap_{k=1}^{n} B_{p, \theta ; k}^{\alpha_{k}}\left(\mathbb{R}^{n}\right) \quad\left(B_{p}^{\alpha_{1}, \ldots, \alpha_{k}} \equiv B_{p, p}^{\alpha_{1}, \ldots, \alpha_{k}}\right) .
$$


In this section we apply Theorem 3.1 to find the asymptotic of the constant in (1.12) as some of the numbers $\alpha_{k}$ tend to 1 .

First we observe that if a function $f \in L^{p}\left(\mathbb{R}^{n}\right)$ has a weak derivative $D_{k} f \in L^{p}\left(\mathbb{R}^{n}\right)$, then

$$
\lim _{\alpha \rightarrow 1-0}(1-\alpha)^{1 / \theta}\|f\|_{b_{p, \theta ; k}^{\alpha}}=\left(\frac{1}{\theta}\right)^{1 / \theta}\left\|D_{k} f\right\|_{p} .
$$

The proof is the same as one given in [15, Proposition 2.5].

Next, we prove the following proposition.

Proposition 4.1. Let $0<\alpha<1, \quad 1 \leq p<\infty$, and $1 \leq k \leq n \quad(n \geq 2)$. Assume that $f \in B_{p ; k}^{\alpha}\left(\mathbb{R}^{n}\right)$. Then $f \in V_{k} \equiv L_{\hat{x}_{k}}^{p}\left[\Lambda_{x_{k}}^{1 / p-\alpha}\right]$ and

$$
\|f\|_{V_{k}} \leq 100[\alpha(1-\alpha)]^{1 / p}\|f\|_{b_{p ; k}^{\alpha}} .
$$

Proof. For a fixed $\hat{x}_{k} \in \mathbb{R}^{n-1}$, denote

$$
f_{\hat{x}_{k}}\left(x_{k}\right)=f\left(x_{k}, \hat{x}_{k}\right), \quad x_{k} \in \mathbb{R} .
$$

For almost all $\hat{x}_{k}$ and any $t>0$ we have by (2.16) and (2.8)

$$
f_{\hat{x}_{k}}^{*}(t)-f_{\hat{x}_{k}}^{*}(2 t) \leq 4 t^{-1 / p} \omega\left(f_{\hat{x}_{k}} ; t\right)_{p} .
$$

It follows that

$$
\left\|f_{\hat{x}_{k}}\right\|_{\Lambda^{\sigma}} \leq 4\left\|f_{\hat{x}_{k}}\right\|_{b_{p, \infty}^{\alpha}(\mathbb{R})}, \quad \sigma=1 / p-\alpha .
$$

Applying Lemma 2.2, we have

$$
\left\|f_{\hat{x}_{k}}\right\|_{b_{p, \infty}^{\alpha}(\mathbb{R})} \leq 8[\alpha(1-\alpha)]^{1 / p}\left\|f_{\hat{x}_{k}}\right\|_{b_{p}^{\alpha}(\mathbb{R})} .
$$

Next, by (2.3),

$$
\left\|f_{\hat{x}_{k}}\right\|_{b_{p}^{\alpha}(\mathbb{R})}^{p} \leq 3^{p} \int_{0}^{\infty} \int_{\mathbb{R}}\left|f\left(x_{k}, \hat{x}_{k}\right)-f\left(x_{k}+h, \hat{x}_{k}\right)\right|^{p} d x_{k} \frac{d h}{h^{\alpha p+1}} .
$$

Integrating with respect to $\hat{x}_{k}$, we obtain

$$
\int_{\mathbb{R}^{n-1}}\left\|f_{\hat{x}_{k}}\right\|_{b_{p}^{\alpha}(\mathbb{R})}^{p} d \hat{x}_{k} \leq 3^{p} \int_{0}^{\infty}\left[h^{-\alpha} \omega_{k}(f ; h)_{p}\right]^{p} \frac{d h}{h} .
$$

Combining this inequality with (4.3) and (4.4), we get (4.2).

Applying Theorem 3.1 and Proposition 4.1, we obtain the following result.

Theorem 4.2. Let $1 \leq p<\infty, n \geq 2 \quad(n \in \mathbb{N})$, and $1 / 2<\alpha_{k}<1(k=1, \ldots, n)$. Assume that

$$
\alpha \equiv n\left(\sum_{k=1}^{n} \frac{1}{\alpha_{k}}\right)^{-1} \leq \frac{n}{p} .
$$

Let $q=n p /(n-\alpha p)$. Then for every function $f \in B_{p}^{\alpha_{1}, \ldots, \alpha_{k}}\left(\mathbb{R}^{n}\right)$ we have that $f \in L^{q, p}\left(\mathbb{R}^{n}\right)$ and

$$
\|f\|_{q, p}^{*} \leq c \prod_{k=1}^{n}\left[\left(1-\alpha_{k}\right)^{1 / p}\|f\|_{b_{p ; k}^{\alpha_{k}}}\right]^{\alpha /\left(n \alpha_{k}\right)},
$$

where $c \equiv c_{n}$ is a constant depending only on $n$. 
Indeed, (3.2), (3.3), and (4.2) immediately yield (4.5) with the constant

$$
c=c_{n}\left[\prod_{k=1}^{n}\left(\frac{\alpha_{k}}{n \alpha_{k}-\alpha}\right)^{\alpha /\left(n \alpha_{k}\right)}\right]^{1 / p} \text {. }
$$

Since $1 / 2<\alpha_{k}<1$ for all $k=1, \ldots, n$, then we have

$$
\frac{\alpha_{k}}{n \alpha_{k}-\alpha} \leq \frac{3}{n} \quad(k=1, \ldots, n)
$$

which implies that $c \leq 3 c_{n}$.

As it was already noted in Introduction, inequality (4.5) follows also from [10, Theorem 1]. Nevertheless, it is much simpler to derive (4.5) from Theorem 3.1.

If $\alpha<n / p$, then by (2.12) and (4.5) we obtain (1.17). In turn, (1.17) and (2.6) imply (1.19) and (1.15).

Assume that for some $k$ there exists a weak derivative $D_{k} f \in L^{p}\left(\mathbb{R}^{N}\right)$. Then for the corresponding term in (4.5) we have by (4.1)

$$
\left(1-\alpha_{k}\right)^{1 / p}\|f\|_{b_{p ; k}^{\alpha_{k}}} \rightarrow\left(\frac{1}{p}\right)^{1 / p}\left\|D_{k} f\right\|_{p}, \quad \alpha_{k} \rightarrow 1 .
$$

We see that (similarly to (3.26)) the embedding $B_{p}^{\alpha_{1}, \ldots, \alpha_{k}}\left(\mathbb{R}^{n}\right) \subset L^{q, p}\left(\mathbb{R}^{n}\right)$ can be split into two parts. The main part is contained in Theorem 3.1. The factors $\left(1-\alpha_{k}\right)^{\alpha /\left(p n \alpha_{k}\right)}$ in (4.5) appear when we apply Proposition 4.1 (i.e., in the "easy" part of (4.5)).

REMARK 4.3. Observe that Maz'ya and Shaposhnikova [19] studied also the behaviour of the optimal constant in (1.13) as $\alpha \rightarrow 0$. More precisely, they proved that the constant in $(1.15)$ can be replaced by

$$
c_{p, n} \alpha(1-\alpha)(n-\alpha p)^{1-p}
$$

It was shown in [15] that this result (as well as (1.15)) follows from the rearrangement estimates obtained in [11]. For anisotropic Besov spaces the asymptotic of the constant in (1.12) in the case when all $\alpha_{k}$ are small can be easily derived from [9, Lemma 5]. Nevertheless, the study of this constant in the general case (when some of the numbers $\alpha_{k}$ tend to 0 and some of them tend to 1 ) requires different arguments. We will present the corresponding result in other paper.

\section{References}

[1] J. Bastero, M. Milman and F. J. Ruiz Blasco, A note on $L(\infty, q)$ spaces and Sobolev embeddings, Indiana Univ. Math. J. 52 (2003), 1215-1230.

[2] C. Bennett, R. DeVore and R. Sharpley, Weak- $L^{\infty}$ and BMO, Ann. of Math. 113 (1981), 601-611.

[3] J. Bourgain, H. Brezis and P. Mironescu, Another look at Sobolev spaces, in: Optimal Control and Partial Differential Equations. In honour of Professor Alain Bensoussan's 60th Birthday. J. L. Menaldi, E. Rofman, A. Sulem (eds.), IOS Press, Amsterdam, 2001, 439-455.

[4] J. Bourgain, H. Brezis and P. Mironescu, Limiting embedding theorems for $W^{s, p}$ when $s \uparrow 1$ and applications, J. Analyse Math. 87 (2002), 77-101. 
[5] H. Brezis, How to recognize constant functions. Connections with Sobolev spaces, Uspekhi Mat. Nauk 57 (2002), no. 4(346), 59-74 (in Russian); English transl.: Russian Math. Surveys 57 (2002), no. 4, 693-708.

[6] E. Gagliardo, Proprietà di alcune classi di funzioni in più variabili, Ricerche Mat. 7 (1958), $102-137$.

[7] M. L. Gol'dman, Embedding of generalized Nikol'skiü-Besov spaces into Lorentz spaces, Trudy Mat. Inst. Steklov 172 (1985), 128-139 (in Russian); English transl.: Proc. Stekolov Inst. Math. 172 (1985), 143-154.

[8] J. Fournier, Mixed norms and rearrangements: Sobolev's inequality and Littlewood's inequality, Ann. Mat. Pura Appl. 148 (1987), 51-76.

[9] V. I. Kolyada, On imbedding in classes $\varphi(L)$, Izv. Akad. Nauk SSSR Ser. Mat. 39 (1975), 418-437 (in Russian); English transl.: Math. USSR Izv. 9 (1975), 395-413.

[10] V. I. Kolyada, On embedding $H_{p}^{\omega_{1}, \ldots, \omega_{\nu}}$ classes, Mat. Sb. 127 (1985), 352-383 (in Russian); English transl.: Math. USSR-Sb. 55 (1986), 351-381.

[11] V. I. Kolyada, Estimates of rearrangements and embedding theorems, Mat. Sb. 136 (1988), 3-23 (in Russian); English transl.: Math. USSR-Sb. 64 (1989), 1-21.

[12] V. I. Kolyada, Rearrangements of functions and embedding theorems, Uspekhi Matem. Nauk 44 (1989), 61-95 (in Russian); English transl.: Russian Math. Surveys 44 (1989), $73-118$.

[13] V. I. Kolyada, On embedding of Sobolev spaces, Mat. Zametki 54 (1993), 48-71 (in Russian); English transl.: Math. Notes 54 (1993), 908-922

[14] V. I. Kolyada, Rearrangement of functions and embedding of anisotropic spaces of Sobolev type, East J. Approx. 4 (1998), 111-199.

[15] V. I. Kolyada and A. K. Lerner, On limiting embeddings of Besov spaces, Studia Math. 171 (2005), 1-13.

[16] E. H. Lieb and M. Loss, Analysis, Grad. Stud. Math. 14, AMS, 1997.

[17] L. H. Loomis and H. Whitney, An inequality related to the isoperimetric inequality, Bull. Amer. Math. Soc. 55 (1949), 961-962.

[18] J. Malý and L. Pick, An elementary proof of sharp Sobolev embeddings, Proc. Amer. Math. Soc. 130 (2001), 555-563.

[19] V. Maz'ya and T. Shaposhnikova, On the Bourgain, Brezis, and Mironescu theorem concerning limiting embeddings of fractional Sobolev spaces, J. Funct. Anal. 195 (2002), 230238.

[20] R. O'Neil, Convolution operators and L(p,q) spaces, Duke Math. J. 30 (1963), 129-142.

[21] J. Peetre, Espaces d'interpolation et espaces de Soboleff, Ann. Inst. Fourier (Grenoble) 16 (1966), 279-317.

[22] S. Poornima, An embedding theorem for the Sobolev space $W^{1,1}$, Bull. Sci. Math. 107 (1983), 253-259.

[23] E. M. Stein and G.Weiss, Introduction to Fourier Analysis on Euclidean Spaces, Princeton Univ. Press, 1971.

[24] P. L. Ul'yanov, Embedding of certain function classes $H_{p}^{\omega}$, Izv. Akad. Nauk SSSR Ser. Mat. 32 (1968), 649-686 (in Russian); English transl.: Math. USSR Izv. 2 (1968), 601-637. 